\title{
Long QT syndrome: Identification of a novel de novo mutation of calmodulin in a newborn girl
}

\author{
Thi Huynh Nga Nguyen ${ }^{1}$, Chi Bao Bui ${ }^{2,3,4}$, Vuong Thao Vy Nguyen ${ }^{2}$, Manh Cong Nguyen ${ }^{2}$, \\ Nguyen Thanh Tung $\mathrm{Vu}^{4}$, Minh Hiep Nguyen ${ }^{5, *}$
}

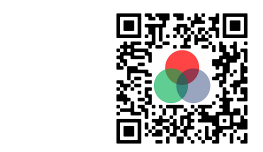

Use your smartphone to scan this QR code and download this article

${ }^{1}$ Department of Biology, Dalat

University, Da Lat City, Viet Nam

${ }^{2}$ Functional Genomics Unit, DNA Medical Technology Company, Ho Chi Minh City, Viet Nam

${ }^{3}$ Center for Molecular Biomedicine, University of Medicine and Pharmacy, Ho Chi Minh City, Viet Nam

${ }^{4}$ Department of Molecular Genetics, City Children's Hospital, Ho Chi Minh City, Viet Nam

${ }^{5}$ Radiation Technology Center, Nuclear Research Institute, Da Lat City, Viet Nam

\section{Correspondence}

Minh Hiep Nguyen, Radiation Technology Center, Nuclear Research Institute, Da Lat City, Viet Nam

Email: jackminhhiep@yahoo.com

\section{History}

- Received: Oct 11, 2021

- Accepted: Dec 25, 2021

- Published: Jan 22, 2022

DOI : 10.15419/bmrat.v9i1.719

\section{Check for updates}

Copyright

(๑) Biomedpress. This is an openaccess article distributed under the terms of the Creative Commons Attribution 4.0 International license.

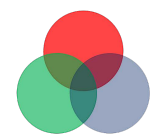

\begin{abstract}
Background: Long QT syndrome (LQTS) is a genetically and phenotypically heterogeneous disorder of ventricular myocardial repolarization that is typically characterized by prolongation of the heart rate-corrected QT interval on a 12-lead electrocardiogram (ECG). LQTS has a complicated etiology since great genotypic and phenotypic overlapping among LQTSs limits the establishment of a diagnosis based solely on clinical features. Case presentation: Here, we describe a Vietnamese newborn with a novel calmodulin (CALM2) variant. The patient was referred to the Department of Cardiology at Children's Hospital 2 in Ho Chi Minh City, Vietnam for multiple clinical cardiac presentations. The patient's family history was negative for cardiovascular diseases; however, she suffered from recurrent syncope and QT interval prolongation on resting in a 12-lead surface ECG. A fetal echocardiogram at 29 weeks detected signs of congenital heart disease. The newborn patient then suffered from sudden cardiac arrest and required cardiopulmonary resuscitation. Two weeks later, the patient suffered from torsade de pointes for the third time and required electrical shocks. An endotracheal tube was placed through the mouth into the trachea to facilitate breathing. Unfortunately, the patient expired at 3 months of age due to serious inflammatory issues. Whole exome sequencing (WES) - a technique used to determine variations in all coding regions (exons) of the known genes - validated a total of four rare variants in four cardiomyopathy (CM) genes, the CALM2, DSP, MYBPC3, and SPEG genes. We identified a novel de novo heterozygous missense mutation, c.280G > T (p.Asp94Tyr), in the CALM2 gene (NM_001305626) encoding calmodulin, which is a ubiquitous calcium-binding protein that is important for a myriad of intracellular signaling events of cardiac function. Conclusion: The results obtained in this study support the "pan-cardiomyopathy panel" approach, in which the molecular diagnosis of LQTS, the early identification of arrhythmia development, and the improved clinical management of cardiovascular disease patients are applied. Trial registration: The procedures were reviewed and approved by the Ethical Committee of the University of Medicine and Pharmacy, Ho Chi Minh City, Vietnam, under the number UMP-VN 2018-10a12.
\end{abstract}

Key words: Calmodulin, Cardiovascular, Long QT syndrome, Mutation, Whole exome sequencing

\section{INTRODUCTION}

Long QT syndrome (LQTS) is a genetically and phenotypically heterogeneous disorder of ventricular myocardial repolarization that is typically characterized by prolongation of the heart rate-corrected QT interval (QTc) on a 12-lead electrocardiogram (ECG) $^{\mathbf{1 , 2}}$. This syndrome leads to torsadogenic syncope, seizures, aborted cardiac arrest, and sudden cardiac death $(\mathrm{SCD})^{3,4}$. LQTS is associated with autopsy-negative sudden death in the young, sudden infant death syndrome (SIDS), and even stillbirths ${ }^{5-7}$. Recently, mutations have been identified in at least 17 LQTS-susceptibility genes ${ }^{\mathbf{8}, 9}$. LQTS has a complicated etiology, including multiple non-genetic and genetic causes ${ }^{10}$. Moreover, LQTS includes the complexity associated with genotype-phenotype correlation because not all mutations on the same gene produce a similar clinical phenotype ${ }^{11}$. The impact on clinical manifestations is unpredictable, as neither the localization of a mutation nor its cellular electrophysiological effect is associated with the phenotypes $^{12}$. In certain cases, different LQTS subtypes have the same cardiac phenotype, and an identical mutation can cause symptomatic and asymptomatic LQTSs and other syndromes, leading to difficulties regarding the management of patients and a requirement for multiple ECG recordings ${ }^{11,13,14}$. Consequently, great genotypic and phenotypic overlapping among LQTSs limits the establishment of a diagnosis based solely on clinical features. Therefore, a specific diagnosis and prognosis for LQTS patients are reliant on next-generation sequencing (NGS) technology and genome-wide association studies. 
NGS refers to high-throughput DNA sequencing technology that generates data on multiple DNA fragments in a single reaction ${ }^{15}$. NGS methods, including whole-exome sequencing (WES) and wholegenome sequencing (WGS), have rapidly become scalable tools that generate a molecular diagnosis for Mendelian genetic disorders ${ }^{15-17}$. The exome, which is composed of exons, comprises approximately $1 \%$ of the total genome and harbors $85 \%$ of interpretable mutations that result in clinical phenotypes ${ }^{18,19}$. Accordingly, the identification and sequencing of this $1 \%$ coding region, which has less repetitive sequences than the noncoding regions, is computationally and analytically less intense ${ }^{15,16}$. WES overcomes the limitations of Sanger sequencing methods because of its speed, cost, and accuracy ${ }^{15-19}$. When used as a first-line test, the diagnostic yield of WES is costeffective and has become a powerful tool in identifying the causal variant(s) for a disease of interest ${ }^{17}$.

Since cardiovascular diseases (CVDs) are highly heritable, human genetic variation is considered to contribute to the detection of individuals who are at risk of developing cardiomyopathy (CM), heart failure, arrhythmias, and vascular diseases.

This present study reports on the family of a newborn girl with multiple clinical cardiac presentations who was diagnosed with LQTS type 3 (as she carried a heterozygous nonsynonymous mutation, c.280G > $\mathrm{T}$ (p.Asp94Tyr), in the calmodulin-2 (CALM2) gene. The results of this study support the genetic etiology for LQTS and highlight the need for screening of the CALM gene in congenital cardiovascular probands.

\section{CASE PRESENTATION}

\section{Materials and methods \\ Patient data}

The present study reports on the Vietnamese family of a newborn index patient who was diagnosed with CVD by specialist doctors at Children's Hospital 2 in Ho Chi Minh City, Viet Nam. The QT intervals were measured on the ECG in lead II or V5, and the QTC was corrected for using Bazett's formula. The LQTS diagnosis was based on the symptoms and QTc.

The clinical manifestation, laboratory results (including ECG) and echocardiography, application of drugs, and prognosis of the patient were reviewed. The parents of the patient provided consent for genetic analysis. The study was approved by the local ethics committee and complied with the standards set by the hospital.

\section{Library preparation and sequencing}

Genomic DNA was isolated from white blood cells using standard techniques (DNA Extraction Kit, Qiagen, Hilden, Germany). The DNA fragments were ligated with adaptors and two paired-end DNA libraries with an insert size of $100-900 \mathrm{bp}$. After enrichment by polymerase chain reaction (PCR), the DNA libraries were prepared for cardiac gene enrichment re-sequencing on a HiSeq 4000 next-generation sequencing system according to the manufacturer's instructions (TruSeq 3000/4000 SBS Kit v3 - protocol HiSeq 3000/4000 system user guide, part \# 15066496, Rev. A HSC 3.3.20). The patient was screened for variants in $142 \mathrm{CM}$-associated genes using the TruSeq ${ }^{\mathrm{TM}}$ Cardiomyopathy gene panel, which resulted in 150-bp paired-end sequencing reads and at least a 100-fold average sequencing depth for each sample.

\section{Alignment and mapping}

The low-quality reads were discarded from raw data to generate clean reads. The clean reads were then aligned to the human reference genome UCSC Genome Build hg19. The Illumina automated workflow was used for the alignment of the reads and base calling: the Burrows-Wheeler Aligner (BWA), Genome Analysis Toolkit (GATK), and Sequence Alignment Map (SAMtools).

\section{Variant annotation and filtering}

For the annotation of variants, we used the ANNOVAR and VEP software programs. The variants were then computationally compared with a list of reported pathogenic variations from the Human Gene Mutation Database (HGMD, professional version). The extremely rare variants located in the exon and consensus splice sequences ( \pm 1 and 2 positions) were filtered using a global minor allele frequency (MAF) of $\leq 0.0005$. The variants that were not in the HGMD and synonymous and intronic variants that were greater than $15 \mathrm{bp}$ from the exon boundaries were not considered.

\section{Variant classification}

The guidelines of the American College of Medical Genetics and Genomics (ACMG) were used for variant classification to assess dominant single-gene mutations with clear clinical impact on CMs (cardiomyopathies). The Exome Aggregation Consortium (ExAC) browser was used as a reference for the allele frequencies. The missense variant was assessed 
using a variety of prediction tools, including deleterious annotation of genetic variants using neural networks (DANN), Genomic Evolutionary Rate Profiling (GERP), MutationTaster, Protein Variation Effect Analyzer (PROVEAN), and Sorting Intolerant from Tolerant (SIFT). The degree of conservation of the affected residue was measured by multiple ortholog alignment using Alamut software (Interactive Biosoftware, Rouen, France).

\section{Sanger sequencing}

All variants of interest were confirmed using Sanger sequencing. The primers for the identified variants were designed using Primer3. The PCR primer pair used to amplify the products were CALM2-F: 5'-CGCCATGTGATGACAAACCT-3' and CALM2R: 5'-GTTCCCTAACAAAGAGCCTC-3'. Direct sequencing of the PCR products was performed in an ABI3130XL sequencer (Applied Biosystems) according to the manufacturer's protocol.

\section{Results}

\section{Patient clinical information}

The subject of this study was a newborn baby girl who suffered from recurrent syncope and QT interval prolongation on a resting 12-lead surface ECG. The patient, who was born to healthy parents, was the family's only child. Her family history was negative for SCD, and both parents were asymptomatic regarding CVD and showed normal ECGs (data not shown).

A fetal echocardiogram at 29 weeks detected signs of congenital heart disease (CHD), including sinus arrhythmia with slowing of the heart rate, macrocardia, and highly inconsistent echocardiographic grading of aortic stenosis (AS) which was represented by bicuspid aortic valve (BAV) disease (Figure $\mathbf{1} \mathbf{A}$ and $\mathbf{B}$ ).

On June 10, 2018, the primigravida mother had a premature rupture of membranes (PROM) at 36 weeks of gestation. After amniorrhexis, a baby girl weighing 2,300 grams, who was in a breech presentation, was delivered in good condition by emergency caesarean section.

The neonate's diagnosis at the time of hospital admission showed a conscious state; however, her sinus rhythm was slow at a rate of 60 beats per minute (bpm).

During hospitalization, the low-birth-weight infant was well breastfed and reached a weight of 2,450 grams after one week. However, the electrocardiography showed a markedly prolonged QTc interval of 700 ms. Sinus arrhythmia was also included in the clinical record as the heart rate ranged from 60 to $100 \mathrm{bpm}$.
Moreover, an inconsistent T wave alternans (TWA) signal was detected using the standard clinical leads. Echocardiograms showed a 2:1 atrioventricular (AV) block, left ventricular non-compaction cardiomyopathy (LVNC) symptoms, and a cardiac ejection fraction (EF) that ranged from 54 to $60.6 \%$ (Figure $\mathbf{1 ~ C}$ and D). Based on these clinical symptoms, the cardiologists strongly suspected LQTS type 3 . The patient was then subdermally implanted with a single-chamber pacemaker beneath the collarbone which was connected to the heart by one wire. A beta-blocker and mexiletine were administered to the patient daily.

On June 29, 2018, the patient suffered from sudden cardiac arrest (SCA) and required cardiopulmonary resuscitation (CPR) for 15 minutes to provide oxygenation and circulation to the body. After this intensive medical care, propranodol was administered to the patient. A low heart rate of 50 to $60 \mathrm{bpm}$ was identified. During the subsequent days, the patient required a single-chamber pacemaker again and was continually medicated with propranodol. The heart rate increased to approximately $70 \mathrm{bpm}$.

Twelve days later, on July 10, 2018, the patient presented to the emergency department due to choking (on milk), cyanosis, and cardiac arrest, and required electrical shock (ES) and respiratory devices. The heart ceased to beat due to torsade de pointes (TdP), which occurred twice and lasted for 3 seconds each time. An ECG Holter monitor showed the occurrence of polymorphic ventricular tachycardia and the heart rate ranged from 80 to $115 \mathrm{bpm}$. A digital Xray image showed cardiac enlargement (Figure 1E). Moreover, the patient's QTc had increased to $720 \mathrm{~ms}$ (Figure 1 F). Thus, the patient was medicated with adrenaline and magnesium sulfate. Two weeks later, the patient suffered from TdP for the third time and required ES and endotracheal intubation (EI). An endotracheal tube was placed through the mouth into the trachea to facilitate breathing. Unfortunately, the patient expired at 3 months of age on September 10, 2018, due to serious inflammatory issues.

\section{Identification of a novel heterozygous CALM2-gene mutation}

Genetic testing could help to increase the diagnostic accuracy and facilitate the clinical assessment and appropriate therapy of patients who have heart failure or arrhythmias, thus, preventing SCD. Therefore, in the present study, WES was performed on the patient to identify genetic variants.

The genetic data obtained for the patient are shown in Table 1. The Illumina sequencer yielded 27,514,276 


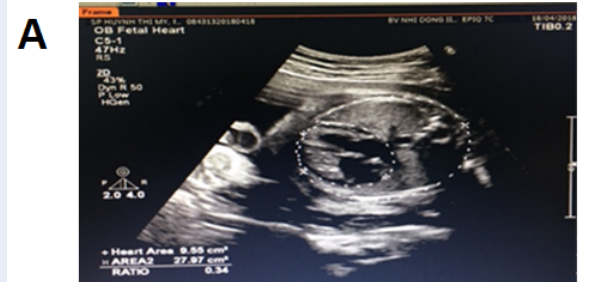

C

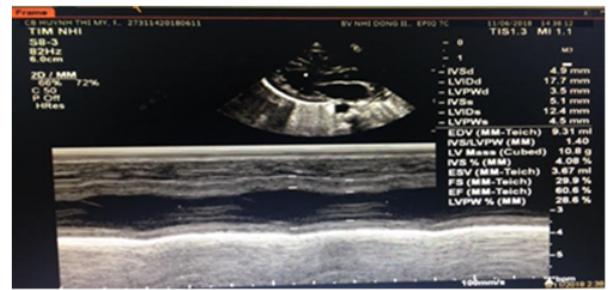

E

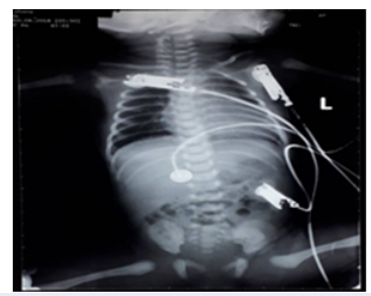

B

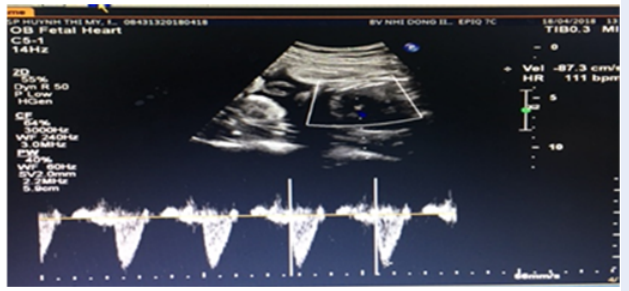

D

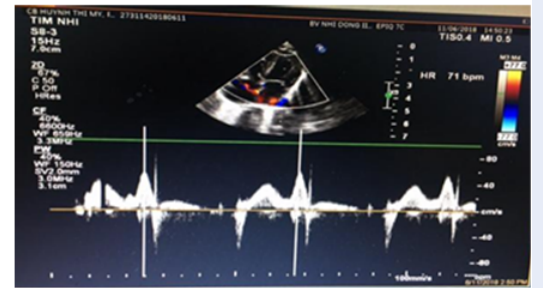

Figure 1: Patient clinical information. A \& B) Fetal echocardiographies at 29 weeks with congenital heart disease signs during the prenatal period. C \& D) Echocardiographies of the patient's first day of birth showed depressed myocardiac function with EF $60.6 \%$ and ventricular abnormalities. E) Digital X-ray image of anteroposterior view of chest showing cardiac morphology with cardiomegaly. F) Electrocardiography showed a markedly prolonged QTc interval of $720 \mathrm{~ms}$. https://doi.org/10.6084/m9.figshare.18865457.v1

\begin{tabular}{lc} 
Table 1: Pre-allignment statistics \\
\hline Total number of reads & $\mathbf{2 7 , 5 1 4 , 2 7 6}$ \\
\hline Average read length (bp) & 151.0 \\
Total yield (Mbp) & 4154 \\
SNP & 88,187 \\
Indel & 182 \\
GC content (\%) & 49 \\
\hline
\end{tabular}

Total yield: (total number of reads) $\mathrm{x}$ (average read length)

Table 2: Statistic variants

\begin{tabular}{lccccc}
\hline No & Gene & Chromosome & Exonic Function & HGVS.c & HGVS.p \\
\hline 1 & CALM2 & 2 & Nonsynonymous SNV & c.G280T & p.D94Y \\
2 & DSP & 6 & Nonsynonymous SNV & c.A3646G & p.I1216V \\
3 & MYBPC3 & 11 & Nonsynonymous SNV & c.C529T & p.R177C \\
4 & SPEG & 2 & Nonsynonymous SNV & c.A8710G & p.T2904A \\
\hline
\end{tabular}

HGVS.c: Variant using HGVS notation (DNA level)

HGVS.p: If variant is coding, this field describes the variant using HGVS notation (protein level) 
A

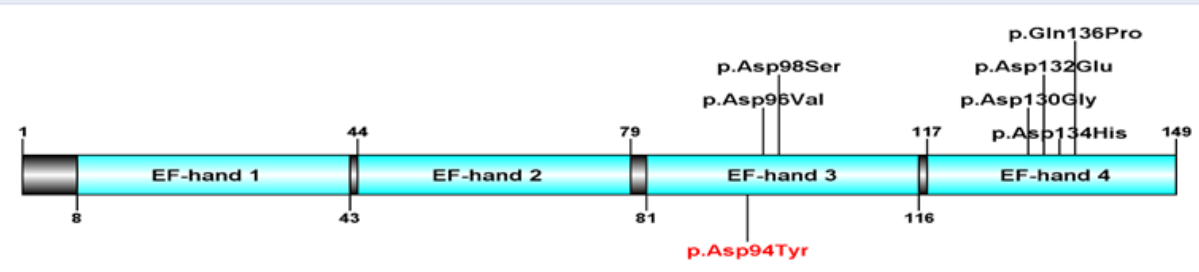

B

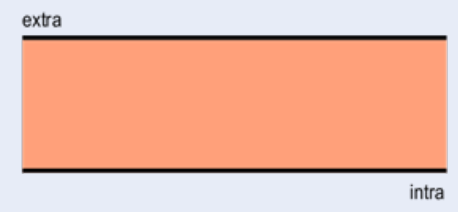

C
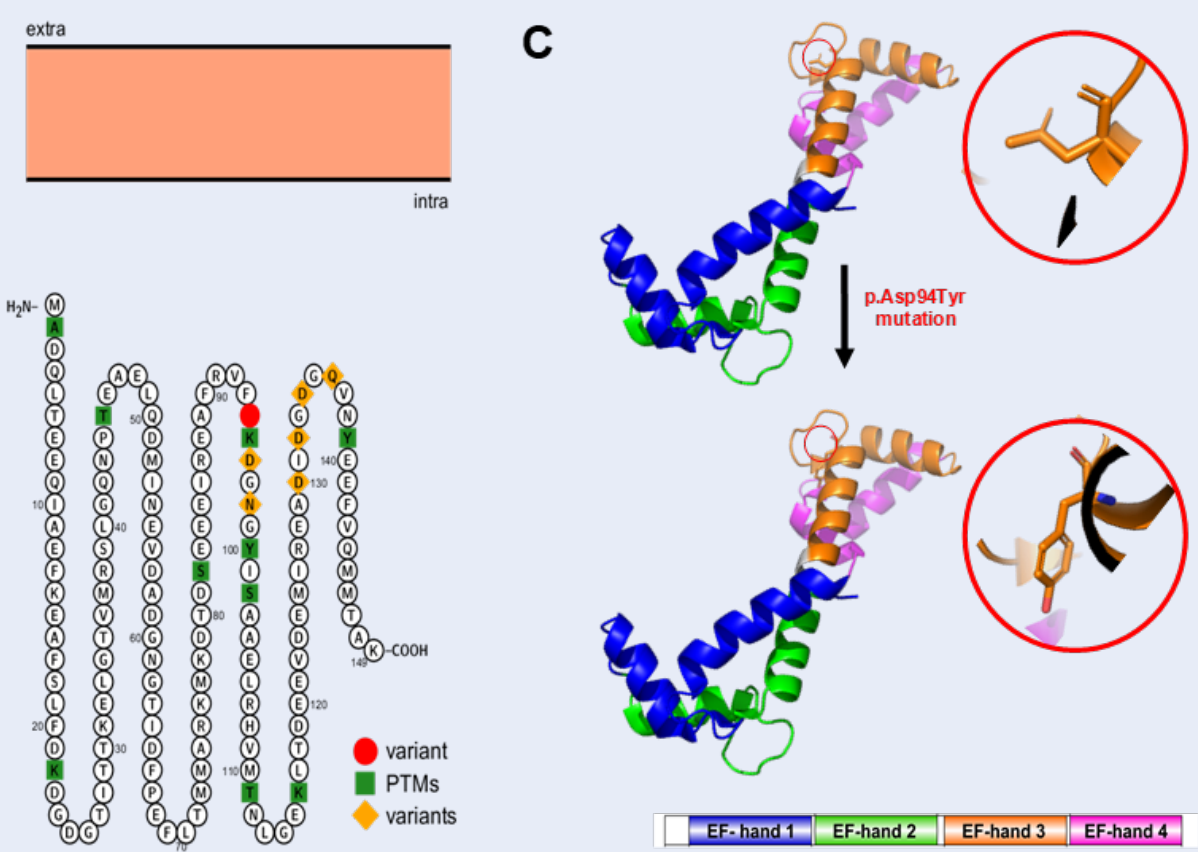

Figure 2: The novel CALM2 mutation. A) Schematic representation of domain structure of calmodulin protein (CaM). CaM, which is encoded by CALM2 gene, is a 21,689-dalton protein of 149 amino acids. CaM is an EF-hand protein composed of two calcium-binding EF-hand motifs (black) in its $\mathrm{N}$-domain and two in its $\mathrm{C}$-domain with a calcium-binding site on each domain (reported by Prosite). Positions of known pathogenic variants over the length of the canonical CaM are shown in black and our novel mutation is shown in red. B) General model of human CaM highlighting its most relevant features. CaM is an intracellular target of the secondary messenger calcium ion. The binding of calcium is required for the activation of CaM. Our novel mutation site is shown in red circle. Several previously known variants are shown in yellow. PTMs: post-translational modifications. The cell membrane is in light orange. The graph was generated using Protter v1.0. C) 3D-structures of CaM model. The positions of codon 94 are circled in red. The structures were created using PyMOL (http://www.pymol.org). https: //doi.org/10.6084/m9.figshare.18865466.v1

reads with an average of $151 \mathrm{bp}$ in length, and the bioinformatics analyses identified a total of 88,187 SNP and 182 Indel. The GC content was $49 \%$.

To further analyze the mutations and identify potential genes, the potential candidates were condensed and included 142 genes that have been linked to CM. These genes were further filtered based on the mapping quality $M Q \geq 40$, prediction of protein alteration, missense variants, and absence from the SNP database of ADS. As a result, four variants in different genes were identified (Table 2).

The results demonstrated that the WES identified a novel heterozygous missense variant in the CALM2 gene (NM_001305626). The variant c.280G > T (p.Asp94Tyr) belonged to the exon 4, where a single nucleotide substitution occurred: $G$ was replaced by $\mathrm{T}$ at position 280 in the cDNA, leading to the replacement of aspartic acid (Asp, D) by tyrosine (Tyr, Y) at the position 94 on the calmodulin protein (CaM) (Figure 2). (p.Asp94Tyr) produces the substitution of an acidic amino acid with a polar non-charged one. The physicochemical properties of Asp and Tyr are very different.

In this case, the only potentially pathogenic variant observed in the patient was c.280G $>\mathrm{T}$ (p.Asp94Tyr) in CALM2 (Table 2). c.280G > T (p.Asp94Tyr) is not 
listed in public databases, nor has it been identified in genotyping projects that included the general population and were comprised of several thousands of subjects (Exome Variant Server, ExAC). The pathogenic effect of the variant (pathogenicity) was graded according to its presence in a previously associated or candidate gene. Therefore, we concluded, given its extremely low MAF, that c.280G > T (p.Asp94Tyr) was a mutation.

The human CaM is a small 149-amino acid protein that comprises two $\mathrm{Ca}^{2+}$-binding lobes at its $\mathrm{N}$ - and C-terminus, each containing two EF-hand structural motifs with $\mathrm{Ca}^{2+}$-binding properties. The novel c.280G > T (p.Asp94Tyr) mutation is located at the central EF-hand calcium-binding 3 domain (Figure $2 \mathrm{~A}$ ). Importantly, the C-terminals EFhands 3 and 4 possess a higher $\mathrm{Ca}^{2+}$-binding affinity than those in the N-terminus and cluster various calmodulinopathy-causing mutations that express multiple arrhythmic phenotypes in the young, suggesting the functional importance of specific topological domains that appear to be intolerant to genetic variation $^{20}$.

To investigate the in silico predicted impact on the calmodulin protein, the 2015 ACMG criteria, as well as widely used software scores, were taken into account. The five protein function prediction bioinformatics tools chosen were DANN, GERP, MutationTaster, PROVEAN, and SIFT. These software programs classified this variant as likely pathogenic (Table 3). Thus, it was extremely likely that this mutation was pathogenic and disease-causing in the patient.

To assess the possibility that the mutation c.280G $>\mathrm{T}$ (p.Asp94Tyr) caused SCD in the proband, WES was also performed on blood samples of the healthy parents (data not shown). Both parents were asymptomatic and did not show phenotypic abnormalities on the baseline or stress test ECGs. Additionally, they both showed a normal resting heart rate in sinus rhythm and normal exercise-induced tachycardia (data not shown). Therefore, we assumed that the mutation was not present in the patient's parents and that the mutation c.280G > T (p.Asp94Tyr) in the CaM was a de novo cause of the SCD in the patient. Consistent with the high-throughput sequencing, the results of the Sanger sequencing revealed that the patient carried a point mutation changing aspartic acid residue (Figure 3B) while her parents did not (Figure $3 \mathrm{C}$ and D). The clinical status was also indicated by the family pedigree of the patient (Figure $3 \mathbf{E}$ ).
The novel mutation c.280G > T (p.Asp94Tyr) altered the highly conserved aspartic acid residue that directly chelates $\mathrm{Ca}^{2+}$ ions in EF-hand domain 3 at position $\mathrm{Y}$ in the pentagonal bipyramidal coordination sphere ${ }^{21}$, predicting a high pathogenic effect. Additionally, the correlation between the patient's symptoms and the known involvement of CaM in the heart $[1,22]$ showed the effect of the mutation on the protein function. Lastly, c.280G > T (p.Asp94Tyr) affects a highly conserved aspartic acid at position 94 . This amino acid mutation point is naturally conservative. Moreover, a comparison of the protein sequence encoded by the CALM2 gene of humans with that of other species indicated that the amino acid of the mutation was not changed (Figure 3 F).

\section{Discussion}

In this study, we attempted to elucidate the genetic basis for LQTS with overlapping features of other CVDs. We evaluated the effectiveness of a new comprehensive NGS CM gene panel assay on a newborn index patient who suffered from CM and demonstrated that it detected mutations with high sensitivity and precision. The technique assisted in establishing a diagnosis in a complex case and amended a diagnosis based solely on clinical features.

The results showed that WES identified a novel heterozygous missense mutation in the CALM2 gene. This gene is located in a $\mathrm{CM}$ susceptibility region (chr2p21) and encodes the CaM protein (phosphorylase kinase, delta), which is an essential signaltransducing protein that regulates calcium-dependent intracellular processes ${ }^{22-25}$. As a calcium sensor protein, calcium-induced CaM modulates the function of cardiac ion channels, including the $\mathrm{Ca}_{V} 1.2$ calcium channel associated with LQTS, the ryanodine receptor (RyR2) associated with catecholaminergic polymorphic ventricular tachycardia (CPVT), and the $\mathrm{Na}_{V} 1.5$ sodium channel ${ }^{26-28}$. Importantly, three CaM genes, including CALM1, CALM2, and CALM3, which have unique nucleotide sequences that all encode for a $100 \%$ identical CaM, are expressed differently in the human heart ${ }^{6,29,30}$. Moreover, the pathogenic variants in these genes have all been associated with early-onset LQTS ${ }^{6,13,20,22,31-36}$. The disease pathogenesis of calmodulinopathic LQTSs depends on (1) the severity of the calcium-binding reduction in the $\mathrm{Ca}^{2+}$ affinity conferred by mutant CaMs, as the LQTS-causative variants reduce CaM affinity to $\mathrm{Ca}^{2+}$ and alter the properties of the cardiac L-type calcium channel (CaV1.2), and (2) the impairment of mutant CaMs in the modulation of the $\mathrm{Na}_{V} 1.5$ and RyR2 ${ }^{1,22}$. 
Table 3: In silico predictionanalysis of the calmodulin variant

\begin{tabular}{llll}
\hline Detected variant & Coding impact & ACMG classification & In silico prediction \\
\hline CALM2:NM_001305626:exo & Nonsynonymous SNV & Likely Pathogenic & DANN (0.9899) \\
& & (PM1, PM2, PM5, PP3) & GERP (5.7399) \\
& & MutationTaster (D) \\
& & PROVEAN (D) \\
& & SIFT (D) \\
\hline
\end{tabular}

SNV: Single Nucleotide Variant, ACMG: The American College of Medical Genetics and Genomics, DANN: Deleterious annotation of genetic variants using neural networks, GERP: Genomic Evolutionary Rate Profiling, PROVEAN: Protein Variation Effect Analyzer, SIFT: Sorting Intolerant from Tolerant, D: Deleterious effect of a variant

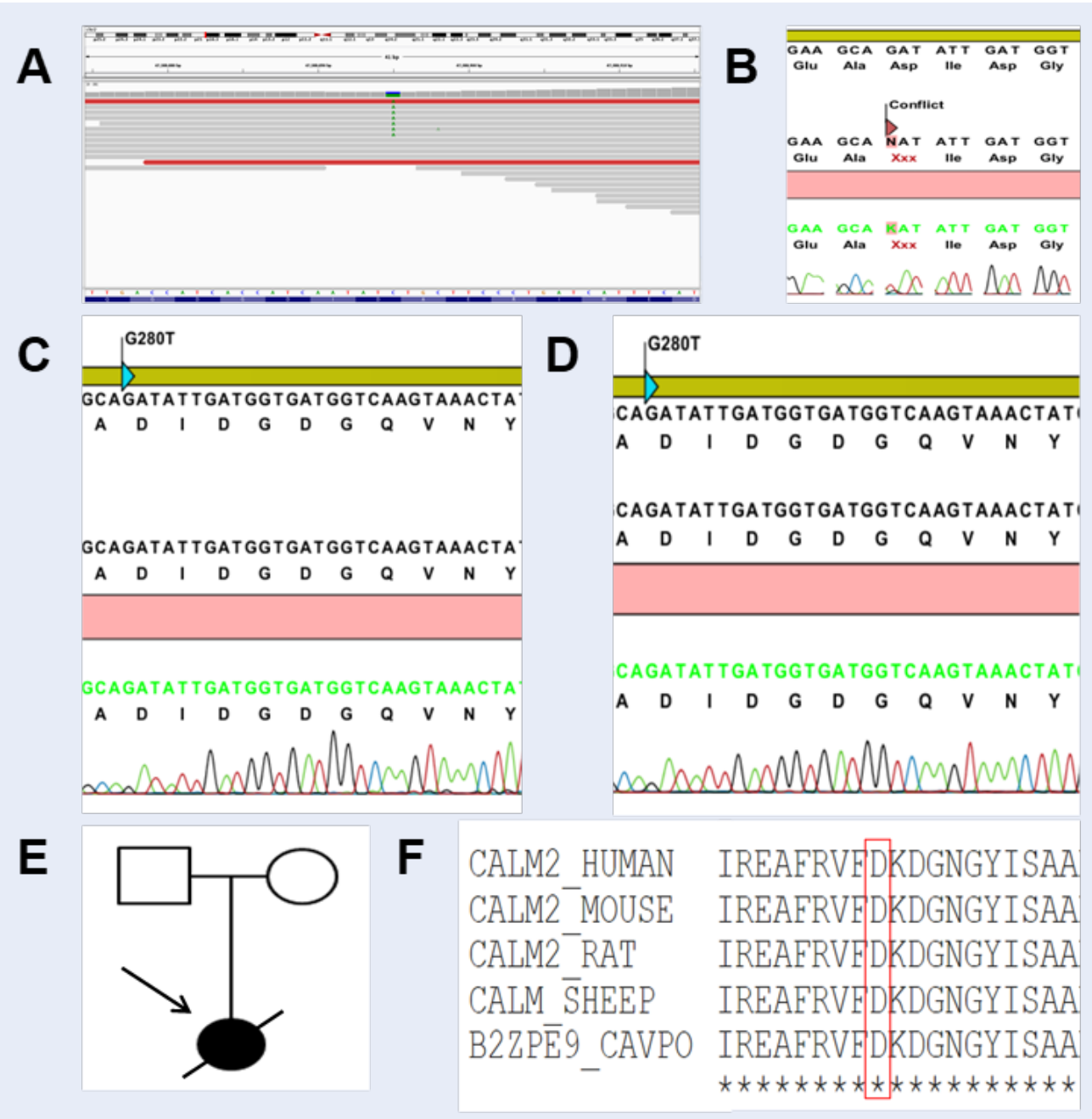

Figure 3: Revealing mutation in the CALM2 gene. A) Nucleotide-level sequence by the BAM file, which is the DNA reverse-complement counterpart showing a previously described missense mutation in the CALM2 gene: c.280G > T (p.(Asp94Tyr)) (detected by the NGS data). B) The mutation was validated by Sanger sequencing. C \& D) Sanger sequencing of CALM2 in father's sample (C) and mother's one (D). E) Patient family pedigree showing clinical status. The proband (marked by the arrow) with filled symbol represents affected individual and the parents with no signs of CVD (empty symbols). F) Protein alignments showing the codon Asp94 conservation across species. https://doi.org/10.6084/m9.figshare.18865490.v1 
Recent advances in WES have shown that approximately $10 \%$ of sporadic severe cases of CCDs are caused by de novo mutations ${ }^{37,38}$. The most recent studies have suggested that de novo mutations in the CALM2 gene lead to severe cardiac arrest in childhood ${ }^{6,13,31,36}$. Three unrelated children aged 4 and 7 had LQTS and CPVT and carried the CALM2 p.Asn98Ser mutation 13,31. An 11-year-old girl who had a CALM2 p.Asp132Gly mutation was reported to suffer from LQTS, sinus bradycardia, and right bundle branch block ${ }^{36}$. A 3-week-old baby girl who had a CALM2 p.Asp96Val mutation showed markedly prolonged QTc and cardiac arrest due to multiple episodes of ventricular fibrillation (VF) ${ }^{6}$. The patient included in the present study who carried the CALM2 p.D94Y mutation and presented with LQTS together with TdP can be added to this list.

The genetic test results of these young patients were suggestive of a hidden channelopathy as well as a molecular diagnosis of calmodulinopathies. Surprisingly, parental genetic screening in various of the patients' families demonstrated that calmodulinopathycausative mutations mostly arose de novo rather than being inherited ${ }^{20}$. Moreover, statistical studies showed that these mutations were single-nucleotide substitutions which led to distinct missense amino acid changes and were mainly distributed in the CALM1 and CALM2 genes ${ }^{20}$. The most important observation is that most of the calmodulinopathycausative mutations were localized in CaM's Cterminal $\mathrm{Ca}^{2+}$-binding EF-hands 3 and 4 domains, in the specific residues directly involved in $\mathrm{Ca}^{2+}$ binding ${ }^{20}$ and predicted a high pathogenic effect. Interestingly, recent studies have shown that the distributions of the mutations in EF-hand 3 motifs, including p.Asp94Ala, p.Asp96His, p.Asp96Val, p.Asn98Ser, p.Asn98Ile, p.Ala103Val, p.Glu105Ala and p.Asp94Tyr (from the present study) all lead to LQTS or other CVDs ${ }^{6,13,32,34,39-42}$.

The findings of the present study reinforce the genetic etiology for LQTS as well as SCD during infancy. Given that mutations in the CaM EF-hands 3 and 4 domains are life-threatening (since these regions directly transduce $\mathrm{Ca}^{2+}$ signals to influence the activity of ion channels, kinases and other target proteins that are essential for cardiac function), a single mutation in the $\mathrm{C}$-domain can reduce the $\mathrm{Ca}^{2+}$ binding affinity up to 53 -fold ${ }^{6}$. Therefore, CALM genes must be screened in congenital LQTS and other CVD probands because timely management may prevent SCD.

\section{CONCLUSIONS}

The present study reports on the family of a newborn girl with multiple clinical cardiac presentations who was diagnosed with LQTS type 3 (the patient carried a heterozygous nonsynonymous mutation, c.280G > $\mathrm{T}$ (p.Asp94Tyr), in the CALM2 gene. This report reinforces the genetic etiology for LQTS and highlights the need for screening of the entire exome in congenital cardiovascular probands.

\section{ABBREVIATIONS}

LQTS: Long QT syndrome; NGS: next-generation sequencing; WES: whole exome sequencing; SCD: sudden cardiac death

\section{ACKNOWLEDGMENTS}

This research is funded by Vietnam National Foundation for Science and Technology Development (NAFOSTED) under grant number 106-YS.012016.39.

We would also like to thank Truong Nhat Vi and Nguyen Minh Tri Viet for the contribution in this work.

\section{AUTHOR'S CONTRIBUTIONS}

Nguyen Thi Huynh Nga and Nguyen Minh Hiep wrote the manuscript. Bui Chi Bao, Nguyen Thi Huynh Nga and Nguyen Minh Hiep designed the study, analyzed and interpreted the data. Bui Chi Bao, Nguyen Vuong Thao Vy, Nguyen Thanh Tung Vu, Nguyen Manh Cong performed the experiments. All authors approved the final manuscript.

\section{FUNDING}

This research is funded by Vietnam National Foundation for Science and Technology Development (NAFOSTED) under grant number 106-YS.012016.39 .

\section{AVAILABILITY OF DATA AND MATERIALS}

All data generated or analyzed during this study are included in this published article.

\section{ETHICS APPROVAL AND CONSENT TO PARTICIPATE}

Procedures were reviewed and approved by the Ethical Committee of the University of Medicine and Pharmacy, Ho Chi Minh, Vietnam. Participant's parents were consenting to including the research methods involved. 


\section{CONSENT FOR PUBLICATION}

Not applicable.

\section{COMPETING INTERESTS}

The authors declare that they have no competing interests.

\section{REFERENCES}

1. Giudicessi JR, Ackerman MJ. Calcium revisited: new insights into the molecular basis of long-QT syndrome. Circulation: Arrhythmia and Electrophysiology. 2016;9(7):e002480. PMID: 27390209. Available from: 10.1161/CIRCEP.116.002480.

2. Moss AJ. Long QT Syndrome. Journal of the American Medical Association. 2003;289(16):2041-4. PMID: 12709446. Available from: $10.1001 /$ jama.289.16.2041.

3. Giudicessi JR, Ackerman MJ. Genotype- and phenotypeguided management of congenital long QT syndrome. Current Problems in Cardiology. 2013;38(10):417-55. PMID 24093767. Available from: 10.1016/j.cpcardiol.2013.08.001.

4. Schwartz PJ, Ackerman MJ. The long QT syndrome: a transatlantic clinical approach to diagnosis and therapy. European Heart Journal. 2013;34(40):3109-16. PMID: 23509228. Available from: 10.1093/eurheartj/eht089.

5. Arnestad M, Crotti L, Rognum TO, Insolia R, Pedrazzini $M$, Ferrandi C. Prevalence of long-QT syndrome gene variants in sudden infant death syndrome. Circulation. 2007;115(3):361-7. PMID: 17210839. Available from: 10.1161/ CIRCULATIONAHA.106.658021.

6. Crotti L, Johnson CN, Graf E, Ferrari GMD, Cuneo BF, Ovadia M. Calmodulin mutations associated with recurrent cardiac arrest in infants. Circulation. 2013;127(9):1009-17. PMID: 23388215. Available from: 10.1161/CIRCULATIONAHA.112. 001216.

7. Tester DJ, Ackerman MJ. Postmortem long QT syndrome genetic testing for sudden unexplained death in the young. Journal of the American College of Cardiology. 2007;49(2):240-6. PMID: 17222736. Available from: 10.1016/j. jacc.2006.10.010.

8. Giudicessi JR, Roden DM, Wilde AA, Ackerman MJ. Classification and reporting of potentially pro-arrhythmic common genetic variation in long QT syndrome genetic testing. Circulation. 2018;137(6):619-30. PMID: 29431662. Available from: 10.1161/CIRCULATIONAHA.117.030142.

9. Schwartz PJ, Ackerman MJ, George AL, Wilde AA. Impact of genetics on the clinical management of channelopathies. Journal of the American College of Cardiology. 2013;62(3):169-80. PMID: 23684683. Available from: 10.1016/j.jacc.2013.04.044.

10. Giudicessi JR, Wilde AA, Ackerman MJ. The genetic architecture of long QT syndrome: A critical reappraisal. Trends in Cardiovascular Medicine. 2018;28(7):453-64. PMID: 29661707. Available from: 10.1016/j.tcm.2018.03.003.

11. Schwartz PJ, Crotti L, Insolia R. Long-QT syndrome: from genetics to management. Circulation: Arrhythmia and Electrophysiology. 2012;5(4):868-77. PMID: 22895603. Available from: 10.1161/CIRCEP.111.962019.

12. Crotti L, Spazzolini C, Schwartz PJ, Shimizu W, Denjoy I, Schulze-Bahr E. The common long-QT syndrome mutation KCNQ1/A341V causes unusually severe clinical manifestations in patients with different ethnic backgrounds: toward a mutation-specific risk stratification. Circulation. 2007;116(21):2366-75. PMID: 17984373. Available from: 10.1161/CIRCULATIONAHA.107.726950.

13. Jiménez-Jáimez J, Doza JP, Ortega A, Macías-Ruiz R, Perin F, del Rey MMRV. Calmodulin 2 mutation N98S is associated with unexplained cardiac arrest in infants due to low clinical penetrance electrical disorders. PLoS One. 2016;11(4):e0153851. PMID: 27100291. Available from: 10. 1371/journal.pone.0153851.
14. Makita N, Behr E, Shimizu W, Horie M, Sunami A, Crotti L. The E1784K mutation in SCN5A is associated with mixed clinical phenotype of type 3 long QT syndrome. The Journal of Clinical Investigation. 2008;118(6):2219-29. PMID: 18451998. Available from: 10.1172/JCI34057.

15. Linthorst GE, Hollak CE. [Whole exome sequencing and whole genome sequencing in undiagnosed disease: of value for certain patient populations]. Nederlands Tijdschrift voor Geneeskunde. 2019;163:3711. PMID: 31120221.

16. Sun Y, Man J, Wan Y, Pan G, Du L, Li L. Targeted next-generation sequencing as a comprehensive test for Mendelian diseases: a cohort diagnostic study. Scientific Reports. 2018;8(1):11646. PMID: 30076350. Available from: 10.1038/s41598-018-30151-

17. Barbitoff YA, Polev DE, Glotov AS, Serebryakova EA, Shcherbakova IV, Kiselev AM. Systematic dissection of biases in whole-exome and whole-genome sequencing reveals major determinants of coding sequence coverage. Scientific Reports. 2020;10(1):2057. PMID: 32029882 Available from: 10.1038/s41598-020-59026-y.

18. Choi M, Scholl UI, Ji W, Liu T, Tikhonova IR, Zumbo P. Genetic diagnosis by whole exome capture and massively parallel DNA sequencing. Proceedings of the National Academy of Sciences of the United States of America. 2009;106(45):19096-101. PMID: 19861545. Available from: 10.1073/pnas.0910672106.

19. Ng SB, Turner EH, Robertson PD, Flygare SD, Bigham AW, Lee C. Targeted capture and massively parallel sequencing of 12 human exomes. Nature. 2009;461(7261):272-6. PMID: 19684571. Available from: 10.1038 /nature08250.

20. Kotta MC, Sala L, Ghidoni A, Badone B, Ronchi C, Parati G. Calmodulinopathy: A novel, life-threatening clinical entity affecting the young. Frontiers in Cardiovascular Medicine. 2018;5:175. PMID: 30574507. Available from: 10.3389/fcvm. 2018.00175 .

21. Chattopadhyaya R, Meador WE, Means AR, Quiocho FA. Calmodulin structure refined at $1.7 \mathrm{~A}$ resolution. Journal of Molecular Biology. 1992;228(4):1177-92. PMID: 1474585. Available from: 10.1016/0022-2836(92)90324-D.

22. Boczek NJ, Gomez-Hurtado N, Ye D, Calvert ML, Tester DJ, Kryshtal D. Spectrum and prevalence of CALM1-, CALM2-, and CALM3-encoded calmodulin (CaM) variants in long QT syndrome (LQTS) and functional characterization of a novel LQTSassociated CaM missense variant, E141G. Circulation: Cardiovascular Genetics. 2016;9:136-6. PMID: 26969752. Available from: 10.1161/CIRCGENETICS.115.001323.

23. Chin D, Means AR. Calmodulin: a prototypical calcium sensor. Trends in Cell Biology. 2000;10(8):322-8. PMID: 10884684 Available from: 10.1016/S0962-8924(00)01800-6.

24. Halling DB, Aracena-Parks P, Hamilton SL. Regulation of voltage-gated $\mathrm{Ca} 2+$ channels by calmodulin. Science\{\&amp;\}\{\#\}×0027;s STKE. 2005;2005(315):re15. PMID: 16369047. Available from: 10.1126/stke.3152005re15.

25. Maier LS, Bers DM, Brown JH. Calmodulin and $\mathrm{Ca} 2+$ /calmodulin kinases in the heart - physiology and pathophysiology. Cardiovascular Research. 2007;73(4):629-30. PMID: 17258179. Available from: 10.1016/j.cardiores.2007.01.005.

26. Gabelli SB, Boto A, Kuhns VH, Bianchet MA, Farinelli F, Aripirala $S$. Regulation of the NaV1.5 cytoplasmic domain by calmodulin. Nature Communications. 2014;5(1):5126. PMID: 25370050. Available from: 10.1038/ncomms6126.

27. Hwang HS, Nitu FR, Yang Y, Walweel K, Pereira L, Johnson $\mathrm{CN}$. Divergent regulation of ryanodine receptor 2 calcium release channels by arrhythmogenic human calmodulin missense mutants. Circulation Research. 2014;114(7):1114-24. PMID: 24563457. Available from: 10.1161/CIRCRESAHA.114. 303391.

28. Levitan IB. It is calmodulin after all! Mediator of the calcium modulation of multiple ion channels. Neuron. 1999;22(4):645-8. PMID: 10230783. Available from: 10.1016/ S0896-6273(00)80722-9. 
29. Fischer R, Koller M, Flura M, Mathews S, Strehler-Page MA, Krebs J. Multiple divergent mRNAs code for a single human calmodulin. The Journal of Biological Chemistry. 1988;263(32):17055-62. PMID: 3182832. Available from: 10.1016/S0021-9258(18)37497-0.

30. SenGupta B, Friedberg F, Detera-Wadleigh SD. Molecular analysis of human and rat calmodulin complementary DNA clones. Evidence for additional active genes in these species. The Journal of Biological Chemistry. 1987;262(34):16663-70. PMID: 2445749. Available from: 10.1016/S0021-9258(18) 49306-4.

31. Fujita S, Nakagawa R, Futatani T, Igarashi N, Fuchigami T, Saito S. Long QT syndrome with a de novo CALM2 mutation in a 4-year-old boy. Pediatrics International. 2019;61(9):852-8. PMID: 31283864. Available from: 10.1111/ped.13959.

32. Makita N, Yagihara N, Crotti L, Johnson CN, Beckmann BM, Roh MS. Novel calmodulin mutations associated with congenital arrhythmia susceptibility. Circulation: Cardiovascular Genetics. 2014;7(4):466-74. PMID: 24917665. Available from: 10.1161/CIRCGENETICS.113.000459.

33. Reed GJ, Boczek NJ, Etheridge SP, Ackerman MJ. CALM3 mutation associated with long QT syndrome. Heart Rhythm. 2015;12(2):419-22. PMID: 25460178. Available from: 10.1016/ j.hrthm.2014.10.035.

34. Takahashi K, Ishikawa T, Makita N, Takefuta K, Nabeshima T, Nakayashiro M. A novel de novo calmodulin mutation in a 6-year-old boy who experienced an aborted cardiac arrest. HeartRhythm Case Reports. 2016;3(1):69-72. PMID: 28491771. Available from: 10.1016/j.hrcr.2016.09.004.

35. Wren LM, Jiménez-Jáimez J, Al-Ghamdi S, Al-Aama JY, Bdeir A, Al-Hassnan ZN. Genetic mosaicism in calmodulinopathy. Circulation Genomic and Precision Medicine. 2019;12(9):37585. PMID: 31454269 . Available from: 10.1161/CIRCGEN.119. 002581 .
36. Zahavich L, Tarnopolsky M, Yao R, Mital S. Novel association of a de novo CALM2 mutation with long QT syndrome and hypertrophic cardiomyopathy. Circulation Genomic and Precision Medicine. 2018;11(10):e002255. PMID: 30354306. Available from: 10.1161/CIRCGEN.118.002255.

37. Homsy J, Zaidi S, Shen Y, Ware JS, Samocha KE, Karczewski KJ. De novo mutations in congenital heart disease with neurodevelopmental and other congenital anomalies. Science. 2015;350(6265):1262-6. PMID: 26785492. Available from: 10.1126/science.aac9396.

38. Zaidi S, Choi M, Wakimoto $H, M a ~ L$, Jiang J, Overton JD. De novo mutations in histone-modifying genes in congenital heart disease. Nature. 2013;498(7453):220-3. PMID: 23665959. Available from: 10.1038 /nature12141.

39. Anderson JH, Tester DJ, Will ML, Ackerman MJ. Whole-exome molecular autopsy after exertion-related sudden unexplained death in the young. Circulation: Cardiovascular Genetics. 2016;9(3):259-65. PMID: 27114410. Available from: 10.1161/ CIRCGENETICS.115.001370.

40. Chaix MA, Koopmann TT, Goyette P, Alikashani A, Latour $F$, Fatah M. Novel CALM3 mutations in pediatric long QT syndrome patients support a CALM3-specific calmodulinopathy. HeartRhythm Case Reports. 2016;2(3):250-4. PMID: 28491681. Available from: 10.1016/j.hrcr.2016.02.002.

41. Daly A, Johnson NM, Decker E, Callis TE, Tahiliani J, Garcia J. Pathogenic variants in calmodulin associated with resuscitated childhood cardiac arrest (Abstract C-AB01-05). Heart Rhythm. 2017;14:2.

42. Gomez-Hurtado N, Boczek NJ, Kryshtal DO, Johnson CN, Sun J, Nitu FR. Novel CPVT-associated calmodulin mutation in CALM3 (CALM3-A103V) activates arrhythmogenic Ca waves and sparks. Circulation: Arrhythmia and Electrophysiology. 2016;9(8):e004161. PMID: 27516456. Available from: 10.1161/ CIRCEP.116.004161. 